\title{
Can progressive resistance training twice a week improve mobility, muscle strength, and quality of life in very elderly nursing-home residents with impaired mobility? A pilot study
}

This article was published in the following Dove Press journal:

Clinical Interventions in Aging

22 April 2013

Number of times this article has been viewed

\section{Lilian Krist ${ }^{\prime}$ \\ Fernando Dimeo² \\ Thomas Keil',3 \\ 'Institute of Social Medicine, Epidemiology, and Health Economics, Charité University Medical Center, ${ }^{2}$ Department of Sports Medicine, Charité University Medical Center, Berlin, ${ }^{3}$ Institute of Clinical Epidemiology and Biometry, University of Wurzburg, Wurzburg, Germany}

Purpose: To determine the effects of progressive resistance training on mobility, muscle strength, and quality of life in nursing-home residents with impaired mobility.

Methods: Nursing-home residents aged 77 years and older with impaired mobility were recruited in Berlin, Germany. The eight-week exercise program consisted of progressive resistance training twice a week. Mobility (primary outcome) was assessed with the Elderly Mobility Scale (zero $=$ worst, $20=$ best) at baseline and after 8 weeks. Muscle strength (secondary outcome) was determined by the eight-repetition maximum. The Short Form-36 Health Survey was used to assess quality of life.

Results: Of the 15 participants (mean age 84 years, range $77-97$ years), ten completed the 8 -week program. Mobility (Elderly Mobility Scale mean \pm standard deviation pre $14.1 \pm 3.2$ and post $17.5 \pm 3.6 ; P=0.005$ ) as well as muscle strength of upper and lower limbs improved (from $62 \%$ at chest press up to $108 \%$ at leg extension machine), whereas most quality of life subscales did not show considerable change.

Conclusion: Resistance training twice a week over 2 months seemed to considerably improve mobility and muscle strength in persons aged 77-97 years with impaired mobility.

Keywords: elderly, resistance training, mobility, muscle strength, nursing home

\section{Introduction}

Advancing age is related to considerable changes in mental and physical health, including loss of muscle mass (sarcopenia) and muscle function. This can lead to impaired physical ability and reduced quality of life. ${ }^{1-3}$ After the age of 70 years, about $1.5 \%$ of muscle mass is lost per year. ${ }^{4}$ Denervation, metabolic, hormonal, or immunological reasons and the reduction of physical activity, in particular, contribute to sarcopenia. ${ }^{5}$ However, muscles can maintain a high degree of plasticity in advanced age, whereas tendons lose their plasticity predisposing them to injuries. ${ }^{6}$ Resistance training may reverse tendon stiffness and reduce the risk of strain injuries. Furthermore, it can increase muscle strength and improve mobility as well as physical functioning in the elderly. ${ }^{7}$

Several studies have investigated exercise programs for the very elderly and found moderate to very high improvements in muscle strength, balance, gait speed, and other outcomes that are indispensable for an independent life.$^{8-10}$ However, most studies examined healthy and community dwelling elderly, ${ }^{11-13}$ whereas persons with impaired physical ability as well as nursing-home residents or persons in long-term care facilities were underrepresented. ${ }^{14}$
Correspondence: Lilian Krist Institute of Social Medicine, Epidemiology, and Health Economics, Charité University Medical Center, 10098 Berlin, Germany

Tel +4930450529 109

Fax +4930450529902

Email lilian.krist@charite.de 
A recent systematic review on the effects of progressive resistance training in elderly nursing-home residents showed that the intensity of the training should be vigorous and the duration at least 2 months. ${ }^{15}$ Most authors describe a frequency of three training sessions per week, but the optimal frequency has not been defined. ${ }^{16-19}$

Therefore, the aim of this study was to investigate how much progressive strength training twice a week over a period of 8 weeks improves mobility, muscle strength, and quality of life in nursing-home residents with impaired mobility aged 75 years and older.

\section{Methods}

\section{Subjects and recruitment}

Between May and July 2009, 34 nursing homes in Berlin were contacted by phone. Out of the twelve that agreed to have a personal meeting with more detailed information, seven homes were excluded due to lack of interest in participation, residents without impaired mobility, or residents were looked after by a legal guardian. Five homes were included in the current study.

Nursing-home residents were included if they fulfilled all of the following criteria: (1) 75 years of age and older; (2) mild to severely impaired mobility defined as an Elderly Mobility Scale (EMS) score between six and 18 points; (3) sufficient language skills; (4) written informed consent; and (5) written consent by their general practitioner. Exclusion criteria were: (1) moderate to severe dementia (MiniMental State Examination $<18$ points, ${ }^{20}$ (2) rheumatologic, orthopedic, or any other condition that could be aggravated by sport; (3) elevated systolic/diastolic blood pressure during exercise $(>230 / 110 \mathrm{mmHg})$; (4) cardiac arrhythmia; (5) epilepsy; or (6) legal care. The ethical review committee of the Charité University Medical Center (Berlin, Germany) approved the study.

\section{Interventions}

Participants underwent a progressive resistance training program twice a week for 8 weeks using the following six gym machines: chest press, rowing machine, and butterfly reverse for the upper limb, leg press and leg extension for the lower limb, and a crunch trainer for the abdominals (gym80 International GmbH, Gelsenkirchen, Germany). Training was performed in three sets of eight repetitions of the individual lifted weight. Between the sets, participants had to pause for at least 1 minute. Total training duration was 45 minutes. At the beginning, all participants received an individual detailed introduction at each gym machine by qualified fitness trainers. The 15 participants were divided into two groups, which trained separately and were supervised by the trainers and the study investigator throughout the program. At each machine, height and angle of the seat were adjusted to the individual needs of the participant. Participants who needed help to get on the seat were supported by the training staff. Sitting height and position as well as velocity and range of movements at each machine were checked throughout the exercise by the trainers. The lifted weight was set by the training staff, documented, and regularly adapted to the augmented muscle strength of participants. As soon as a participant could lift the weight more than eight times in a row in all of the three sets, the weight was increased to the next level. Depending on the machines, weight increments ranged from 5-10 kg. Every deviation of regular training was documented. Water was offered to participants ad libitum.

\section{Outcomes}

Primary outcome of the study was the change in mobility determined by the standardized and validated EMS, a sevenitem instrument with a good sensitivity and practicability, assessed by the study physician. The score ranges from zero (minimal mobility) to 20 (maximal mobility)..$^{21-23}$

Secondary outcomes were changes in muscle strength and quality of life. They were measured before and after the training intervention. Muscle strength was measured indirectly by documenting the respective lifted weight. For the assessment of quality of life, the Short Form-36 Health Survey (SF-36) was used. ${ }^{24}$

All participants underwent a short medical exam including a resting electrocardiogram in the Department of Sports Medicine at the Charité University Medical Center. In the gym, the trainers determined the individual eight-repetition maximum, defined as the highest weight that a participant can lift a maximum of eight times in a row. It is comparable to $80 \%$ of the one-repetition maximum (the highest weight a person can lift only once). The latter was not used due to a higher risk of causing blood pressure peaks in comparison to the eight-repetition maximum. During this introductory exercise, blood pressure was measured after every weightlifting series at each machine to detect potential blood pressure peaks.

All participants received a free 3-monthly membership for the gym including insurance, which covered possible accidents occurring during the stay at the gym. 
During transportation from the nursing home to the gym, they were insured by a private transportation company.

\section{Statistical analysis}

This study was planned as a pilot study without a priori sample size estimation. A pre-post comparison of assessed outcomes (mean \pm standard deviation) was carried out by the Wilcoxon signed-rank test for paired samples. The alpha-type error was set at 0.05 , with a power of $80 \%$. Data analysis was conducted with SPSS 12.0.0 (SPSS Inc, Chicago, IL, USA). The outcome variables mobility and muscle strength were assessed as continuous variables from zero (minimum) to 20 (maximum EMS score) and lifted weight in kilograms, respectively. Quality of life was measured as a continuous variable from zero to 100 points.

\section{Results}

\section{Recruitment and baseline characteristics}

Fifteen nursing-home residents aged 77-97 years (mean age 84 years) were recruited. In the current analysis, ten participants who completed the 8-week program were included: four men (mean age 88 years, range $80-95$ years) and six women (mean age 81 years, range $77-87$ years). During the course of the 8 weeks, three men and two women dropped out of the study for the following reasons: back pain, which was not related to the intervention; depression; insufficient adjustment of insulin therapy to the exercise program; broken foot (at the nursing home); ripped catheter during changing of clothes before the training. The five participants who dropped out were comparable in age, sex, and body mass index to the ten participants who completed the 8 weeks of intervention, with an average of 15/16 completed sessions (Table 1).

\section{Outcomes}

After the 8 -week intervention, mobility improved by $24 \%$ $(P=0.005$; Table 2). The effect was larger in men compared to women (27\% versus $21 \%$ ): baseline EMS scores of 14 versus 18 and 15 versus 18 after 8 weeks for men and women, respectively. Mobility increased in every individual participant (Figure 1). Muscle strength (eight-repetition maximum) increased at every machine (Table 2 ). At the rowing machine and the leg extension machine, the lifted weight was doubled. The number of sit-up repetitions increased fourfold (pre $10.5 \pm 3.1$, post $41.7 \pm 12.1$ repetitions; $P=0.027$ ). Women showed a higher improvement in the eight-repetition maximum, particularly at the rowing machine and leg extension (Figure 2).
Table I Baseline characteristics of participants and dropouts $(\mathrm{n}=15)$

\begin{tabular}{lll}
\hline Variable & $\begin{array}{l}\text { Participants } \\
(\mathbf{n}=1 \mathbf{0})\end{array}$ & $\begin{array}{l}\text { Dropouts } \\
(\mathbf{n}=\mathbf{5})\end{array}$ \\
\hline Sex & & \\
$\quad$ Men $(\mathrm{n})$ & 4 & 2 \\
$\quad$ Women (n) & 6 & 3 \\
Age, years & $84.1 \pm 5.7$ & $84.6 \pm 7.4$ \\
Anthropometry & & \\
$\quad$ Body weight, kg & $71.6 \pm 14.8$ & $66.3 \pm 13.0$ \\
$\quad$ Body mass index, $\mathrm{kg} / \mathrm{m}^{2}$ & $25.8 \pm 5.1$ & $24.7 \pm 4.1$ \\
Mental status & & \\
$\quad$ Cognitive function: score of $\mathrm{MMSE}^{\dagger}$ & $27 \pm 3$ & $26 \pm 3$ \\
$\quad$ Depression: score of GDS ${ }^{\ddagger}$ & $8 \pm 5$ & $12 \pm 7$ \\
\hline
\end{tabular}

Notes: $\$ Maximum: 30 points; ${ }^{0}-5$ indicates normal mood, $6-11$ indicates mild, and 12-30 indicates manifest depression; data represents mean \pm standard deviation unless otherwise stated.

Abbreviations: GDS, Geriatric Depression Scale; MMSE, Mini-Mental State Examination.

After the 8-week training program, quality of life did not change considerably regarding both the physical and emotional sum scales of the SF-36: pre 30/100, post 30/100 and pre 59/100, post 56/100 $(P=0.29)$, respectively. There were also no relevant improvements regarding the subscales of the SF-36 except for the subscale of physical functioning, which slightly improved (pre 27/100, post 32/100; $P=0.54$ ).

\section{Discussion Main findings}

This pilot study represents an approach to the development of an exercise program focused on the elderly. The progressive resistance program with two sessions per week over a period of 8 weeks improved mobility and muscle strength in nursing-home residents over 75 years of age with impaired mobility. Overall quality of life did not change after the 2-month program.

\section{Comparison with other studies}

This study showing that progressive resistance training increases muscle strength confirmed results from previous studies such as the randomized controlled trial by Ferri et al who trained participants aged $65-81$ years at $80 \%$ of the onerepetition maximum with knee extension machines. The onerepetition maximum increased as well as the cross-sectional muscle area. ${ }^{25}$ Rosendahl et al showed positive effects of 3 months resistance training (eight to twelve-repetition maximum) in participants with a mean age of 84 years, which were still present after 6 months. ${ }^{26}$

Resistance training programs for nursing-home residents with a high-intensity program (eight-repetition maximum or 
Table 2 Changes in mobility and muscle strength in ten participants who completed the resistance training program

\begin{tabular}{|c|c|c|c|c|c|}
\hline \multirow[t]{2}{*}{ Variable } & \multirow[t]{2}{*}{ Baseline } & \multirow[t]{2}{*}{8 weeks } & \multirow[t]{2}{*}{$P$-value } & \multicolumn{2}{|c|}{$\begin{array}{l}\text { Mean of difference } \\
\text { ( } 8 \text { weeks versus baseline) }\end{array}$} \\
\hline & & & & Absolute & $\%$ \\
\hline \multicolumn{6}{|l|}{ Mobility } \\
\hline Score on Elderly Mobility Scale & $14.2 \pm 3.4$ & $17.5 \pm 3.6$ & 0.005 & $3.3 \pm 0.9$ & $24 \% \pm 8 \%$ \\
\hline \multicolumn{6}{|c|}{ Muscle strength (eight-repetition maximum) } \\
\hline Chest press, kg & $17.0 \pm 7.9$ & $27.5 \pm 10.6$ & 0.005 & $10.5 \pm 5.0$ & $62 \%$ \\
\hline Rowing machine, $\mathrm{kg}$ & $17.0 \pm 9.8$ & $33.5 \pm 12.0$ & 0.005 & $16.5 \pm 7.1$ & $97 \%$ \\
\hline Butterfly reverse, kg & $14.5 \pm 6.4$ & $24.5 \pm 10.9$ & 0.008 & $10.0 \pm 7.0$ & $74 \%$ \\
\hline Leg press, $\mathrm{kg}^{\dagger}$ & $35.2 \pm 15.4$ & $63.7 \pm 25.9$ & 0.007 & $28.4 \pm 15.0$ & $81 \%$ \\
\hline Leg extension, $\mathrm{kg}$ & $13.0 \pm 7.5$ & $27.0 \pm 10.6$ & 0.005 & $14.0 \pm 6.1$ & $108 \%$ \\
\hline
\end{tabular}

Notes: ${ }^{\dagger}$ Only nine participants could perform this exercise; data represents mean \pm standard deviation; bold indicates statistical significance (alpha-type error set at 0.05$)$.

$80 \%$ of the one-repetition maximum), as used in the current study, seem to be the most effective method to increase muscle strength..$^{15,17-19,26,27}$ However, the strong positive effect in muscle strength improvement shown in the current study was in accordance with only one of these studies, ${ }^{17}$ whereas the other studies showed smaller effects on muscle strength. ${ }^{18,19,26}$

Regarding training frequency, sessions offered three times a week were used most often in studies focusing on the elderly. ${ }^{14,15}$ In contrast to this approach, the current study examined a training program with only two sessions per week and was able to show even stronger effects than in comparable studies. ${ }^{27,28}$

Training duration did not seem to have a comparable influence as training intensity. Latham et al showed an effect size difference of 0.15 between short ( $\leq 12$ weeks) and long training duration ( $>12$ weeks) in 41 trials with 1955 participants aged 60 years and over. ${ }^{14}$ As in the current study, most interventions had a duration of $8-12$ weeks. ${ }^{18,19,27,29}$

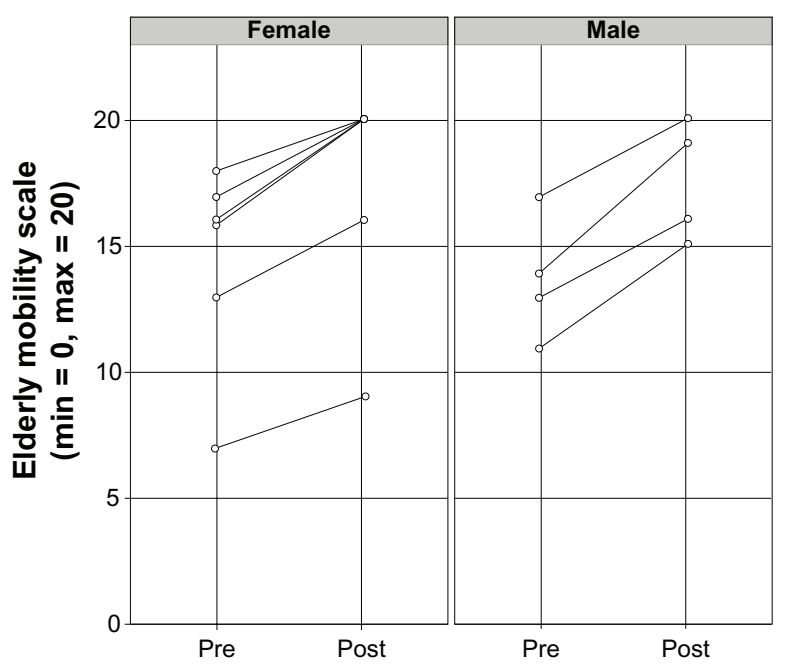

Figure I Individual improvement of mobility score on the Elderly Mobility Scale before and after training intervention for women $(n=6)$ and men $(n=4)$.
Progressive resistance training improves not only muscle strength and mobility but also physical abilities, including simple and more complex daily activities in the elderly. However, there is insufficient evidence on long-term effects as concluded by the authors of a Cochrane review. ${ }^{30}$

Overall quality of life, shown with the physical and emotional sum scale of the SF-36, did not improve in the current study. It is assumed that the 8-week duration of the intervention was too short to detect considerable changes in quality of life. Furthermore, the structure of the SF-36 may have been too complex for this age group (eg, heterogeneity of answering categories). Interviews had to be conducted with some participants who were not able to read the questionnaire by themselves.

A shorter questionnaire with simpler answering categories such as the SF-12 may be an alternative to assess quality of life in future studies with the elderly. ${ }^{31}$

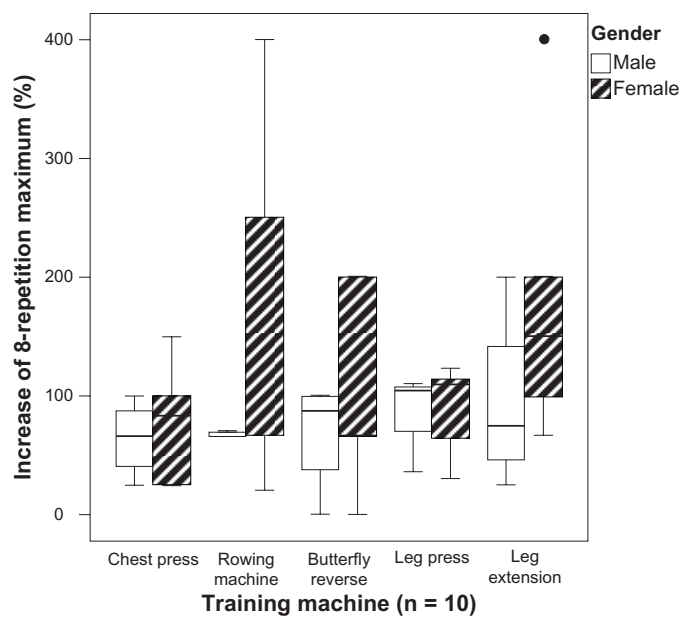

Figure 2 Comparison of percentage increase in eight-repetition maximum for each training machine for male $(n=4)$ and female $(n=6)$ study participants. Notes: Horizontal line in the box represents the median; lower and upper end of the box represents the 25th and 75th percentile, respectively; maximum length of each whisker represents 1.5 times the interquartile range; dot represents the outlier. 


\section{Strengths and limitations}

A particular feature of this study was the investigation of very elderly nursing-home residents with impaired mobility who have hardly been included in intervention studies as shown by the review of Valenzuela. ${ }^{15}$ A strength of this study was the use of validated instruments like the EMS and the SF-36..$^{22,24}$

Several potential limitations have to be considered when interpreting the results of this pilot study. Due to the lack of a control group, the possibility that other factors in addition to the resistance program contributed to the improved mobility and muscle strength cannot be ruled out. Furthermore, familiarization with the gym equipment may have contributed to the training effect, since most participants were not used to resistance training in a gym. The small study population of this investigation cannot be considered as representative for nursing-home residents in Berlin. Most contacted homes were not interested in an exercise program for residents with impaired mobility. For safety and legal considerations, several inclusion and exclusion criteria had to be defined in order not to put participants at risk and aggravate preexisting diseases. Persons under legal care (about $80 \%$ of nursinghome residents) were not eligible and were therefore not included in the study. Although several study participants dropped out, two-thirds completed the 8-week intervention, showing the feasibility of this resistance program in persons up to almost 100 years of age with impaired mobility.

\section{Conclusion}

A progressive resistance training program only twice a week over a period of 8 weeks seems to be a beneficial intervention to improve mobility and muscle strength in nursing-home residents with impaired mobility aged up to 97 years. The intervention did not seem to influence quality of life as assessed by the SF-36 over the 2-month study period; however, it cannot be excluded that a longer intervention may have beneficial effects on quality of life as well.

Randomized controlled trials evaluating the benefits of resistance training in frail nursing-home residents are urgently needed. In these trials, the setting should be adapted and implementable in the daily routine of the elderly regarding intensity, duration, and frequency of the training; special attention should be paid to a presumably high dropout rate due to multimorbidity.

\section{Acknowledgments}

The authors thank all study participants and "McFit" for providing the gym and equipment as well as their trainers.
Costs of transportation, facilities, trainers, and training clothes for the participants were provided by McFit. Authors would also like to thank Felix Fischer for participating in the graph preparation.

\section{Disclosure}

The authors report no conflicts of interest in this work.

\section{References}

1. Imagama S, Hasegawa Y, Matsuyama Y, et al. Influence of sagittal balance and physical ability associated with exercise on quality of life in middle-aged and elderly people. Arch Osteoporosis. 2011;6(1-2): 13-20.

2. Metter EJ, Conwit R, Tobin J, Fozard JL. Age-associated loss of power and strength in the upper extremities in women and men. J Gerontol A Biol Sci Med Sci. 1997;52(5):B267-B276.

3. Stenholm S, Harris TB, Rantanen T, Visser M, Kritchevsky SB, Ferrucci L. Sarcopenic obesity: definition, cause, and consequences. Curr Opin Clin Nutr Metab Care. 2008;11(6):693-700.

4. Vandervoort AA, McComas AJ. Contractile changes in opposing muscles of the human ankle joint with aging. JAppl Physiol. 1986;61(1): 361-367.

5. Doherty TJ. Invited review: aging and sarcopenia. J Appl Physiol. 2003; 95(4):1717-1727.

6. Narici MV, Reeves ND, Morse CI, Maganaris CN. Muscular adaptations to resistance exercise in the elderly. J Musculoskelet Neuronal Interact. 2004;4(2):161-164.

7. Reeves ND. Adaptation of the tendon to mechanical usage. J Musculoskelet Neuronal Interact. 2006;6(2):174-180.

8. Pijnappels M, Reeves ND, Maganaris CN, van Dieen JH. Tripping without falling; lower limb strength, a limitation for balance recovery and a target for training in the elderly. J Electromyogr Kinesiol. 2008;18(2): 188-196.

9. Kerse N, Peri K, Robinson E, et al. Does a functional activity programme improve function, quality of life, and falls for residents in long term care? Cluster randomised controlled trial. BMJ. 2008;337: a1445.

10. Katula JA, Rejeski WJ, Marsh AP. Enhancing quality of life in older adults: a comparison of muscular strength and power training. Health Qual Life Outcomes. 2008;6:45.

11. Alfieri FM, Riberto M, Abril-Carreres A, et al. Effectiveness of an exercise program on postural control in frail older adults. Clin Interv Aging. 2012;7:593-598.

12. Bulat T, Hart-Hughes S, Ahmed S, et al. Effect of a group-based exercise program on balance in elderly. Clin Interv Aging. 2007;2(4):655-660.

13. Zech A, Drey M, Freiberger E, et al. Residual effects of muscle strength and muscle power training and detraining on physical function in community-dwelling prefrail older adults: a randomized controlled trial. BMC Geriatr. 2012;12:68.

14. Latham NK, Bennett DA, Stretton CM, Anderson CS. Systematic review of progressive resistance strength training in older adults. J Gerontol A Biol Sci Med Sci. 2004;59(1):48-61.

15. Valenzuela T. Efficacy of progressive resistance training interventions in older adults in nursing homes: a systematic review. J Am Med Dir Assoc. 2012;13(5):418-428.

16. Venturelli M, Lanza M. Positive effects of physical training in activity of daily living-dependent older adults. Exp Aging Res. 2010;36(2): 190-205.

17. Fiatarone MA, Marks EC, Ryan ND, Meredith CN, Lipsitz LA, Evans WJ. High-intensity strength training in nonagenarians. Effects on skeletal muscle. JAMA. 1990;263(22):3029-3034.

18. Sullivan DH, Wall PT, Bariola JR, Boop MM, Frost YM. Progressive resistance muscle strength training of hospitalized frail elderly. $\mathrm{Am} J$ Phys Med Rehabil. 2001;80(7):503-509. 
19. Seynnes O, Fiatarone Singh MA, Hue O, Pras P, Legros P, Bernard PL. Physiological and functional responses to low-moderate versus highintensity progressive resistance training in frail elders. $J$ Gerontol A Biol Sci Med Sci. 2004;59(5):503-509.

20. Folstein MF, Folstein SE, McHugh PR. "Mini-mental state.” A practical method for grading the cognitive state of patients for the clinicans. J Psychiatr Res. 1975;12(3):189-198.

21. Spilg EG, Martin BJ, Mitchell SL, Aitchison TC. A comparison of mobility assessments in a geriatric day hospital. Clin Rehabil. 2001; 15(3):296-300.

22. Yu MS, Chan CC, Tsim RK. Usefulness of the Elderly Mobility Scale for classifying residential placements. Clin Rehabil. 2007;21(12): 1114-1120.

23. de Morton NA, Berlowitz DJ, Keating JL. A systematic review of mobility instruments and their measurement properties for older acute medical patients. Health Qual Life Outcomes. 2008;6:44.

24. Ware JE Jr, Sherbourne CD. The MOS 36-item short-form health survey (SF-36). I. Conceptual framework and item selection. Med Care. 1992;30(6):473-483.

25. Ferri A, Scaglioni G, Pousson M, Capodaglio P, Van Hoecke J, Narici MV. Strength and power changes of the human plantar flexors and knee extensors in response to resistance training in old age. Acta Physiol Scand. 2003;177(1):69-78.
26. Rosendahl E, Lindelof N, Littbrand H, et al. High-intensity functional exercise program and protein-enriched energy supplement for older persons dependent in activities of daily living: a randomised controlled trial. Aust J Physiother. 2006;52(2):105-113.

27. Fiatarone MA, O'Neill EF, Ryan ND, et al. Exercise training and nutritional supplementation for physical frailty in very elderly people. N Engl J Med. 1994;330(25):1769-1775.

28. Serra-Rexach JA, Bustamante-Ara N, Hierro Villaran M, et al. Short-term, light- to moderate-intensity exercise training improves leg muscle strength in the oldest old: a randomized controlled trial. J Am Geriatr Soc. 2011;59(4):594-602.

29. Pu CT, Johnson MT, Forman DE, et al. Randomized trial of progressive resistance training to counteract the myopathy of chronic heart failure. J Appl Physiol. 2001;90(6):2341-2350.

30. Liu C, Latham NK. Progressive resistance strength training for improving physical function in older adults [review]. Cochrane Database Syst Rev. 2009;3:CD002759.

31. Ware J Jr, Kosinski M, Keller SD. A 12-item short-form health survey: construction of scales and preliminary tests of reliability and validity. Med Care. 1996;34(3):220-233.
Clinical Interventions in Aging

\section{Publish your work in this journal}

Clinical Interventions in Aging is an international, peer-reviewed journal focusing on evidence-based reports on the value or lack thereof of treatments intended to prevent or delay the onset of maladaptive correlates of aging in human beings. This journal is indexed on PubMed Central, MedLine, the American Chemical Society's 'Chemical Abstracts

\section{Dovepress}

Service' (CAS), Scopus and the Elsevier Bibliographic databases. The manuscript management system is completely online and includes a very quick and fair peer-review system, which is all easy to use. Visit http://www.dovepress.com/testimonials.php to read real quotes from published authors. 